\title{
The Expression of E-Cadherin and Vimentin in Non-Small-Cell Lung Cancer and Their Correlation with Epithelial-Mesenchymal Transition (EMT)
}

\author{
Mingyuan Tian', $\mathbf{Y u ~ L i}^{2 *}$ \\ ${ }^{1}$ Department of Endocrinology, The Second Affiliated Hospital, Chongqing Medical University, Chongqing, China \\ ${ }^{2}$ Department of Pathology, Institute of Neuroscience, Chongqing Medical University, Chongqing, China \\ Email: mingyuant@163.com, *liyu100@163.com
}

How to cite this paper: Tian, M.Y. and Li, Y. (2018) The Expression of E-Cadherin and Vimentin in Non-Small-Cell Lung Cancer and Their Correlation with Epithelial-Mesenchymal Transition (EMT). Journal of Biosciences and Medicines, 6 , 1-8.

https://doi.org/10.4236/jbm.2018.65001

Received: March 26, 2018

Accepted: May 18, 2018

Published: May 21, 2018

\begin{abstract}
To investigate the expression of E-cadherin and Vimentin protein in non-small-cell lung cancer (NSCLC) and to analyze the relationship between their expression and epithelial-mesenchymal transition (EMT). Immunochemical staining with SP method was conducted to detect the protein expression of E-cadherin and Vimentin in 44 lung cancer tissues. E-cadherin was highly expressed in all normal lung tissues, and Vimentin was lowly expressed in all normal lung tissues. The expression rate of E-cadherin and Vimentin was $40.9 \%$ and $25.0 \%$ in NSCLC cases. The expressions of E-cadherin and Vimentin were not associated with gender, age, histopathological grading, lymphatic metastasis or smoking status $(\mathrm{P}>0.05)$. But they were associated with clinical stage $(\mathrm{P}<0.05)$. In addition, a negative correlation between the E-cadherin and Vimentin was observed (Spearman's $r=-0.397, p<0.05$ ). E-cadherin and Vimentin are involved in the transformation of epithelial cells into the mesenchymal cells, which play important role in the metastasis of lung cancer.
\end{abstract}

\section{Keywords}

Lung Cancer, E-Cadherin, Vimentin, EMT

\section{Introduction}

Lung cancer is one of the most common therioma, and lung cancer is the main cause of cancer death worldwide. Metastasis is considered the critical factor in clinical treatment of tumor. However, the molecular and cellular mechanism of 
tumor metastasis is not well understood. Several studies showed the epithelial-mesenchymal transition (EMT) was involved in the tumor metastasis. EMT is a physiologic or pathologic process that allows phenotypic alteration of cells change from an epithelial to a mesenchymal phenotype and makes cancer cells to obtain invasive and metastatic ability [1]. E-cadherin and Vimentin are well known for their alternative expression and peculiar roles in EMT, and they are vital bio-markers representing epithelial cells and mesenchymal cells respectively [2]. Pathologists confirmed that EMT consisted in mammary tumor because they found reduced expression of E-cadherin [3] and over-expression of Vimentin [4] in the research of breast cancer. The aim of our study was to evaluate the expression of E-cadherin and Vimentin in cases of lung cancer, to better comprehend their relationships in EMT and to address their potential roles on the development of lung cancer.

\section{Materials and Methods}

\subsection{Clinical Samples}

44 patients with NSCLC had been diagnosed during period from 2009 to 2010 at the Department of Pathology, the First Affiliated Hospital of Chongqing Medical University were enrolled into the study: 27 patients without lymphatic metastasis and 16 patients with lymphatic metastasis. Among all the cases, 21 were squamous cell carcinomas, 20 adenocarcinomas and 2 adenosquamous carcinomas. The age of patients ranges from 23 to 85 . All patients had undergone surgical resection, but no one received chemotherapy and radiotherapy.

\subsection{Methods}

Specimens obtained by surgical resection were fixed in $10 \%$ buffered formalin and paraffin embedded. These formalin-fixed, paraffin-embedded tissues were sectioned at $5 \mu \mathrm{m}$ thickness for standard immunohistochemical staining. The slides were deparaffinized in xylene two times, and then hydrated using graded ethanol before submitted for antigen retrieval. Briefly, antigen retrieval was carried out by microwave pretreatment in sodium citrate ( $\mathrm{pH} \mathrm{6.0)} \mathrm{for} 15 \mathrm{~min}$; endogenous peroxidase activity was quenched by immersing slides in distilled water, containing $3.0 \%$ hydrogen peroxide for $15 \mathrm{~min}$. After antigen retrieval, the slides were washed three times for $5 \mathrm{~min}$ each. Nonspecific antigen sites were blocked with normal 5\% goat serum for $1 \mathrm{~h}$. Then the sections were incubated with a mouse monoclonal E-cadherin anti-body (ready-made, MAB-0589 maixin bio, Fuzhou, China) or mouse monoclonal Vimentin anti-body (ready-made, MAB-0178, maixin bio, Fuzhou, China) over-night at $4^{\circ} \mathrm{C}$. Slides were washed in phosphat-buffered saline.and then incubated with a biotinylated horse anti mouse secondary antibody (Co Win Biotech, Beijing, China) for $60 \mathrm{~min}$ at room temperature.

Antigen-antibody complexes were detected with the Avidin-biotn peroxidase for $60 \mathrm{~min}$ at room temperature. Bound antibody was visualized with DAB (Co 
Win Biotech, Beijing, China). Nuclei was counter-stained with hematoxylin. Negative controls were carried out by substituting the primary antibody with PBS.

Evaluation of Immunohistochemical Staining

Immunostained sections were assessed by two pathologists independently. The positive staining of Vimentin was in the cytoplasm, while the positive of E-cadherin was in the membrane. The results were expressed according to semiquantitative rules: staining intensity:0, no staining; 1) weak staining; 2) moderate staining; 3) intense staining; Scoring of the tissue microarray: 0) less than $10 \%$ staining; 1 ) more than $10 \%$, less than $25 \%$ staining; 2 ) more than $26 \%$, less than $50 \%$ staining; 3 ) more than $51 \%$, less than $75 \%$ staining; 4 ) more than $76 \%$ staining. The scores for both parameters were combined for each sample to obtain a final score.

\subsection{Statistical Analysis}

SPSS for windows (version 19.0) was used for statistical analyses. Correlation among different variables (gender, age, tumor primary site, lymphatic metastasis, stage at diagnosis, and smoking status) was assayed by the X2 test and Fisher's exact test. Spearman's model was used to investigate the relationship between E-cadherin and Vimentin. Statistical significance was declared if the p-value was $<0.05$.

\section{Results}

The E-cadherin expression in NSCLC was 40.9\% (18/44). The expression of E-cadherin was not associated with gender, age, histopathological grading or smoking status $(\mathrm{P}>0.05)$, but was closely related to different clinical stages and lymphatic metastasis $(\mathrm{P}<0.05)$ (Table 1 , Figure 1$)$. The expression of Vimentin in lung cancer was $25.0 \%(11 / 44)$.

The expression of Vimentin was not associated with gender, age, histopathological grading or smoking status $(\mathrm{P}>0.05)$, but was closely related to different clinical stages and lymphatic metastasis $(\mathrm{P}<0.05)$ (Table 1 , Figure 1$)$.

The expression levels of E-cadherin and Vimenin were analyzed by Spearman's correlation test. Significant negative correlation were found between the expression of E-cadherin and Vimentin $(r=-0.397, \mathrm{p}<0.05)($ Table 2$)$.

\section{Discussion}

In recent years, the incidence and the mortality of lung cancer has been increasing constantly. It is important to understand the molecular and cellular mechanisms of lung cancer in order to improve therapeutic outcome. EMT is considered to be involved in tumor progression, which can alter the phenotype of tumor cells and increase their ability of invasion and migration [2]. Cell phenotype change quickly and convertibly and epithelial cells lose adhesion junctions, modulating their polarity and change their cytoskeleton in EMT [5]. The EMT 
Table 1. The expression of E-cadherin and Vimentin and their associations with clinicopathologic parameters.

\begin{tabular}{|c|c|c|c|c|c|c|c|}
\hline \multirow{2}{*}{ characteristics } & \multirow{2}{*}{$\mathrm{n}$} & \multicolumn{3}{|c|}{ E-cadherin $(+)$} & \multicolumn{3}{|c|}{ Vimentin (+) } \\
\hline & & $\mathrm{n}$ & $\mathrm{X}^{2}$ & P-value & $\mathrm{n}$ & $\mathrm{X}^{2}$ & P-value \\
\hline \multicolumn{8}{|l|}{ Age (year) } \\
\hline$<60$ & 24 & 10 & 0.013 & 0.911 & 8 & 1.956 & 0.162 \\
\hline$\geq 60$ & 20 & 8 & & & 3 & & \\
\hline \multicolumn{8}{|l|}{ Histology } \\
\hline AdC & 21 & 10 & 0.389 & 0.533 & 5 & 0.030 & 0.862 \\
\hline SCC & 21 & 8 & & & 5 & & \\
\hline $\mathrm{AC}$ & 2 & 0 & & & 1 & & \\
\hline \multicolumn{8}{|l|}{ Sex } \\
\hline Male & 30 & 14 & 1.293 & 0.256 & 8 & 0.140 & 0.709 \\
\hline Female & 14 & 4 & & & 3 & & \\
\hline Smoking & 25 & 11 & 0.229 & 0.632 & 7 & 0.278 & 0.598 \\
\hline Non-smoking & 19 & 7 & & & 4 & & \\
\hline \multicolumn{8}{|l|}{ LYM metastasis } \\
\hline Yes & 17 & 3 & 6.201 & 0.013 & 7 & 3.866 & 0.049 \\
\hline No & 27 & 15 & & & 4 & & \\
\hline \multicolumn{8}{|l|}{ Stage } \\
\hline I-II & 32 & 16 & 4.011 & 0.045 & 4 & 9.778 & 0.002 \\
\hline III-IV & 12 & 2 & & & 7 & & \\
\hline
\end{tabular}

AdC: adenocarcinoma; SCC: squamous cell carcinoma; AC: adenosquamous carcinoma LYM metastasis: lymphatic metastasis.

Table 2. Relations between expression of protein e-cadherin and vimentin.

\begin{tabular}{|c|c|c|c|}
\hline \multirow[b]{2}{*}{ E-cadherin } & \multicolumn{2}{|c|}{ Vimentin } & \multirow{2}{*}{ sum } \\
\hline & - & + & \\
\hline- & 15 & 10 & 25 \\
\hline+ & 18 & 1 & 19 \\
\hline sum & 33 & 11 & 44 \\
\hline
\end{tabular}

was firstly defined during heart morphogenesis, the notion was extended to the formation of mesoderm and neural crest later [6]. EMT involves three main characters: a) loss or decrease of epithelial cell adhesion (some adhesion molecule) and of polarized cytoskeleton; b) change of cytokeratin of epithelial cell. c) improved cell invasion and migration. There are many proteins involving EMT, such as Cadherin, $\beta$-Catenin, Paxillin, Vimentin, slug and so on [3]. E-cadherin and Vimentin are well konwn for their selective expression in epithelial and mesenchymal cellular states respectively. 

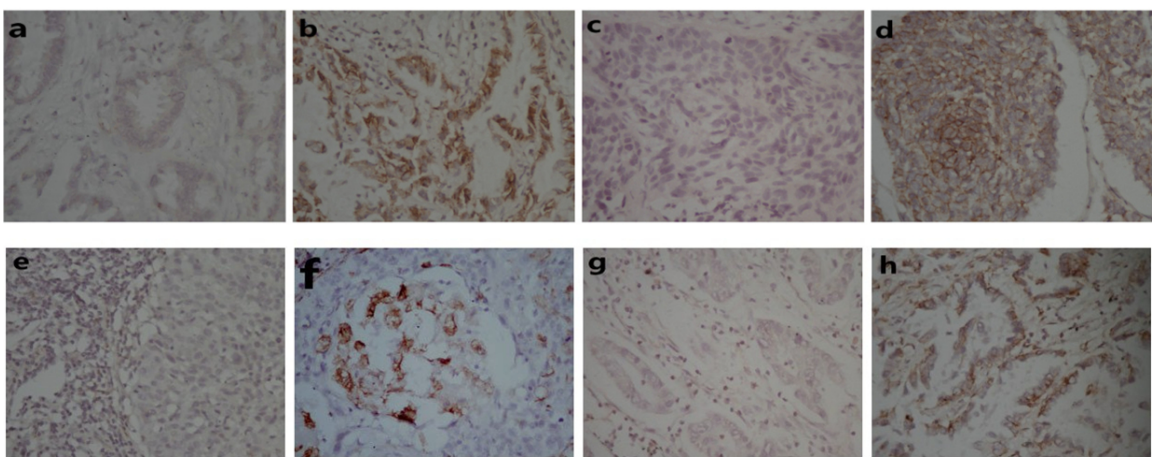

Figure 1. The staining of E-cadherin in lung cancer. Expression of E-cadherin and Vimentin in NSLCL. (a) The negative membranous staining of E-cadherin in adenocarcinoma of lung cancer ( $\times 400)$; (b) The positive membranous staining of E-cadherin in adenocarcinoma of lung cancer $(\times 400)$; (c) The negative membranous staining of E-cadherin in squamous carcinoma of lung cance $(\times 400)$; (d) The positive membranous staining of E-cadherin in squamous carcinoma of lung cancer $(\times 400)$; (e) The negative cytoplasmic staining of Vimentin in squamous carcinoma of lung cancer $(\times 400)$; (f) The positive cytoplasmic staining of Vimentin in squamous carcinoma of lung cancer $(\times 400)$. (g) The negative cytoplasmic staining of Vimentin in adenocarcinoma of lung cancer ( $\times 400)$; (h) The positive cytoplasmic staining of E-cadherin in adenocarcinoma of lung cancer $(\times 400)$.

E-cadherin is a transmembrane glycoprotein that mediates calcium-dependent cell-cell adhesion, expresses in the epithelial cells mostly and involves in the connects of cells extensively. E-cadherin is well regarded to remain the integrity of epithelial cells and to play an important role in differentiation of cells. Menwhile, it was reported that decreased expression of E-cadherin triggered the EMT and played vital role in invasion of cancer [7]. Vlahov $\mathrm{N}$ et al. [8] found the expression of E-cadherin lost mostly or partially in some malignant tumor deriving from epithelial tissues. The evidence showed that the E-cadherin lost or decreased in a range of tumors, such as breast cancer [3], lung cancer [9], pancreas cancer [10].

In our study, the positive expression rate of E-cadherin was about $41.9 \%$, the negative expression rate of that was nearly $58.1 \%$, which was consistent to the result reported by Stefanou D [11]. Lee YC et al. [12] showed that E-cadherin expression were higher in tumors with early stage, well differentiated and non-invasion. Meanwhile, E-cadherin expression was lower in tumors that were in late stage, poor differentiated and high invasion [13]. In our study, the normal expression rate $(16.7 \%)$ of E-cadherin in advanced stage is meaningfully lower than that $(51.6 \%)$ in primary stage $(\mathrm{p}=0.037)$, which was also observed by others. It suggests that adhesion of tumor cells decrease and accelerate EMT as E-cadherin reduces in the progression of tumor. Prudkin L et al. [14] found low E-cadherin expression were statistically higher in squamous cell carcinoma than in adenocarcinoma of lung cancer. But in our research, we found there was no statistical correlation between expression of E-cadherin and histopathlogical grading. 
Vimentin is a kind of cytoskeletal proteins, which consist in endothelial cells, fibroblasts, leukocytes and so on. It is absent in normal epithelial cells and involved in EMT. Sutoh, Yoneyama M et al. [15] showed Vimentin was up-regulated in bladder cancer. Other studies showed that the over-expression of Vimentin was associated with high invasiveness and poor prognosis in hepatocellular carcinoma [16]. In our study, the expression rate of Vimentin was $25.6 \%$ in lung cancer. We found that the over-expressed rate of Vimentin in advanced stage $(58.3 \%)$ was significantly higher than that in primary stage $(12.9 \%$, $\mathrm{p}=0.002$ ). Our finding showed the over-expression of Vimentin was not associated with in gender, age, histopathological grading and smoking status, but was related to TNM tumor stage.

Our study found E-cadherin had negative correlation with Vimentin, and decreased E-cadherin immunohistochemical expression was accompanied by $\mathrm{Vi}$ mentin up-regulation in late stage, which was consistent with previous research that EMT was involved in the progression of tumor [17].

In addition, the expression rate of E-cadherin was 55.6\% and that of Vimentin was $14.8 \%$ in cases without lymphatic metastasis, and the rate of them were $17.6 \%$ and $41.2 \%$ respectively in cases with lymphatic metastasis, so our results suggest that lymphatic metastasis has increased epithelial-to-mesenchymal transition expression compared with primary tumors.

Meanwhile, Dasgupta P et al. [18] showed nicotine, an active component of cigarettes, had been found to cause down-regulation of E-cadherin and up-regulation of Vimentin in lung cancer cell line A549 in vitro. The aged, smoking male tend to suffer lung squamous carcinoma from the standpoint of clinical medicine. However, we found no correlation between E-cadherin and sex, age and smoking.

In conclusion, in our study, we evaluated the expression of two proteins related to EMT phenotype throughout the progression of NSCLC, and discussed the expression of E-cadherin and Vimentin in NSCLC for clinical features to better understand the importance of EMT in the pathogenesis and progression of lung cancer.

\section{Acknowledgements}

We are sincerely gratitude to the patients enrolled in our program, their extraordinary cooperation and support makes these studies become possible.

\section{References}

[1] Singh, R., Mandhani, A., Agrawal, V. and Garg, M. (2018) Positive Correlation between Matrix Metalloproteinases and Epithelial-to-Mesenchymal Transition and Its Association with Clinical Outcome in Bladder Cancer Patients. Cancer Microenviron. https://doi.org/10.1007/s12307-017-0199-4

[2] Zielinska, H.A., Holly, J.M.P., Bahl, A. and Perks, C.M. (2018) Inhibition of FASN and ERalpha Signalling during Hyperglycaemia-Induced Matrix-Specific EMT Promotes Breast Cancer Cell Invasion via a Caveolin-1-Dependent Mechanism. 
Cancer Lett.

[3] Kashiwagi, S., Asano, Y., Goto, W., Takada, K., Takahashi, K., Hatano, T., Tanaka, S., Takashima, T., Tomita, S., Motomura, H., Ohsawa, M., Hirakawa, K. and Ohira, M. (2018) Mesenchymal-Epithelial Transition and Tumor Vascular Remodeling in Eribulin Chemotherapy for Breast Cancer. Anticancer Res, 38, 401-410.

[4] Bollong, M.J., Pietila, M., Pearson, A.D., Sarkar, T.R., Ahmad, I., Soundararajan, R., Lyssiotis, C.A., Mani, S.A., Schultz, P.G. and Lairson, L.L. (2017) A Vimentin Binding Small Molecule Leads to Mitotic Disruption in Mesenchymal Cancers. Proc Natl Acad Sci U S A 114 E9903-E9912. https://doi.org/10.1073/pnas.1716009114

[5] King, L.E., Love, C.G., Sieber, O.M., Faux, M.C. and Burgess, A.W. (2016) Differential RNA-Seq Analysis Comparing APC-Defective and APC-Restored SW480 Colorectal Cancer Cells. Genom Data, 7, 293-296.

https://doi.org/10.1016/j.gdata.2016.02.001

[6] Thiery, J.P., Acloque, H. Huang, R.Y. and Nieto, M.A. (2009) Epithelial-Mesenchymal Transitions in Development and Disease. Cell, 139, 871-890. https://doi.org/10.1016/j.cell.2009.11.007

[7] Schmalhofer, O., Brabletz, S. and Brabletz, T. (2009) E-Cadherin, Beta-Catenin, and ZEB1 in Malignant Progression of Cancer. Cancer Metastasis Rev, 28, 151-166. https://doi.org/10.1007/s10555-008-9179-y

[8] Vlahov, N., Scrace, S., Soto, M.S., Grawenda, A.M., Bradley, L., Pankova, D., Papaspyropoulos, A., Yee, K.S., Buffa, F., Goding, C.R., Timpson, P., Sibson, N. and O’Neill, E. (2015) Alternate RASSF1 Transcripts Control SRC Activity, E-Cadherin Contacts, and YAP-Mediated Invasion. Curr Biol, 25, 3019-3034. https://doi.org/10.1016/j.cub.2015.09.072

[9] Rhys, A.D., Monteiro, P., Smith, C., Vaghela, M., Arnandis, T., Kato, T., Leitinger, B., Sahai, E., McAinsh, A., Charras, G. and Godinho, S.A. (2018) Loss of E-Cadherin Provides Tolerance to Centrosome Amplification in Epithelial Cancer Cells. J Cell Biol, 217, 195-209. https://doi.org/10.1083/jcb.201704102

[10] Tian, L., Lu., Z.P., Cai, B.B., Zhao, L.T., Qian, D., Xu, Q.C., Wu, P.F., Zhu, Y., Zhang, J.J., Du, Q., Miao, Y. and Jiang, K.R. .(2016) Activation of Pancreatic Stellate Cells Involves an EMT-Like Process. Int J Oncol, 48, 783-792. https://doi.org/10.3892/ijo.2015.3282

[11] Chelidonis, G., Kavantzas, N., Patsouris, E., Pagaki, E., Athanasiadou, A.M., Agrogiannis, G. and Athanasiadou, P. (2009) DNA Ploidy, E-Cadherin, Beta-Catenin Expression and Their Clinicopathologic Significance in Imprints of Non-Small Cell Lung Cancer. Anal Quant Cytol Histol, 31, 332-339.

[12] Lee, Y.C., Wu, C.T., Chen, C.S., Hsu, H.H. and Chang, Y.L. (2002) The Significance of E-cadherin and Alpha-, Beta-, and Gamma-Catenin Expression in Surgically Treated Non-Small Cell Lung Cancers of $3 \mathrm{~cm}$ or Less in Size. J Thorac Cardiovasc Surg, 123, 502-507. https://doi.org/10.1067/mtc.2002.119334

[13] Lee, Y.C., Wu, C.T., Chen, C.S. and Chang, Y.L. (2000) E-Cadherin Expression in Surgically-Resected Non-Small Cell Lung Cancers-A Clinicopathological Study. Thorac Cardiovasc Surg, 48, 294-299. https://doi.org/10.1055/s-2000-7885

[14] Prudkin, L., Liu, D.D., Ozburn, N.C., Sun, M., Behrens, C., Tang, X., Brown, K.C., Bekele, B.N., Moran, C. and Wistuba, I.I. (2009) Epithelial-to-Mesenchymal Transition in the Development and Progression of Adenocarcinoma and Squamous Cell Carcinoma of the Lung. Mod Pathol, 22, 668-678. https://doi.org/10.1038/modpathol.2009.19

[15] Sutoh, Yoneyama, M., Hatakeyama, S., Habuchi, T., Inoue, T., Nakamura, T., 
Funyu, T., Wiche, G., Ohyama, C. and Tsuboi, S. (2014) Vimentin Intermediate Filament and Plectin Provide a Scaffold for Invadopodia, Facilitating Cancer Cell Invasion and Extravasation for Metastasis. Eur J Cell Biol, 93, 157-169.

https://doi.org/10.1016/j.ejcb.2014.03.002

[16] Fan, L.C., Shiau, C.W., Tai, W.T., Hung, M.H., Chu, P.Y., Hsieh, F.S., Lin, H., Yu, H.C. and Chen, K.F. (2015) SHP-1 Is a negative Regulator of Epithelial-Mesenchymal Transition in Hepatocellular Carcinoma. Oncogene, 34, 5252-5263. https://doi.org/10.1038/onc.2014.445

[17] Tsoukalas, N., Aravantinou-Fatorou, E., Tolia, M., Giaginis, C., Galanopoulos, M., Kiakou, M., Kostakis, I.D., Dana, E., Vamvakaris, I., Korogiannos, A., Tsiambas, E., Salemis, N., Kyrgias, G., Karameris, A. and Theocharis, S. (2017) Epithelial-Mesenchymal Transition in Non Small-Cell Lung Cancer. Anticancer Res, 37, 1773-1778. https://doi.org/10.21873/anticanres.11510

[18] Wang, W., Liu, F., Wang, C., Wang, C., Tang, Y. and Jiang, Z. (2017) Glutathione S-Transferase A1 Mediates Nicotine-Induced Lung Cancer Cell Metastasis by Promoting Epithelial-Mesenchymal Transition. Exp Ther Med, 14, 1783-1788.

https://doi.org/10.3892/etm.2017.4663 